\title{
Neuroendocrine Carcinoma of the Breast is More Aggressive Than Breast Invasive Ductal Carcinoma: A Propensity Score Matching Study Based on SEER Database
}

\section{Yu-Qiu Chen}

affilited Jinling Hospital, medical school of Nanjing University

Yu Xue

Southeast University

Xiao-Fan Xu

affilited Jinling Hospital, medical school of Nanjing University

Xu-Lin Wang

affilited Jinling Hospital, medical school of Nanjing University

Li-Qun Huo

affilited Jinling Hospital, medical school of Nanjing University

Lu Wang

affilited Jinling Hospital, medical school of Nanjing University

Tian-Yu Di

Nanjing Medical University

Jia-Wei Xu

Nanjing Medical University

Jun Gu ( $\nabla$ gujunjiangsu@outlook.com )

affiliated Jinling Hospital,Medical school of Nanjing University

Guo-hua Zhou

affilited Jinling Hospital, medical school of Nanjing University

Research article

Keywords: Neuroendocrine carcinoma, invasive ductal carcinoma, SEER, prognosis

Posted Date: October 5th, 2020

DOl: https://doi.org/10.21203/rs.3.rs-74221/v1

License: (c) (1) This work is licensed under a Creative Commons Attribution 4.0 International License.

Read Full License 
Page $2 / 13$ 


\section{Abstract}

Background Neuroendocrine carcinoma (NEC) of the breast is a very rare malignant tumor whose clinic pathological features and prognosis are not well defined. The aim of this study was to compare the clinic pathological features and clinical outcome between breast neuroendocrine carcinoma and breast invasive ductal carcinoma (IDC) and to know the independent prognostic risk factors of NEC.

Method Patients with breast NEC and breast IDC were identified through the Surveillance, Epidemiology, and End Results (SEER) database from 2010 to 2015. Then we compared the clinic pathological features and outcome between the two cohorts. Propensity score matching (PSM) was performed to balance the effects of baseline clinic pathological differences. Potential prognostic factors of breast NEC were identified by the univariate and multivariate Cox analysis.

Results In total, we identified 208 patients with breast NEC and 275,878 cases with breast IDC.

Compared with IDC, NEC was significantly correlated with higher stage, larger tumor size, more distant transfers and no population of HER2 enriched subtype. There were significant differences on the choice of treatment therapy between NEC and IDC ( $p=0.000)$. More patients chose not to undergo surgery $(35.1 \%$ vs. $9.0 \%$ ), and fewer patients chose breast conserving surgery than IDC patients (32.2\% vs. $51.1 \%$ ). Compared with IDC patients, NEC patients were more likely to choose chemotherapy $(p=0.039)$ and more conservative in radiotherapy $(\mathrm{p}=0.000)$. The 66 -month overall survival (OS) rate in NEC and IDC was $44.5 \%$ and $80.2 \%$ before PSM and was $49.8 \%$ and $71.4 \%$ after PSM, respectively. The 66 -month breast cancer specific survival (BCSS) rate in NEC and IDC was $56.3 \%$ and $88.8 \%$ before PSM and was $54.1 \%$ and $80.5 \%$ after PSM, respectively. Finally, we identified independent prognostic factors, age, stage, pathological type, distant metastasis, chemotherapy, and molecular typing.

Conclusions Breast NEC has distinct clinic pathological features and a significantly worse clinical outcome than common IDC.

\section{Background}

Primary mammary neuroendocrine carcinoma (NEC) is a rare histological cancer with a reported prevalence of $2-5 \%{ }^{1}$. Neuroendocrine breast neoplasms in the 5 th Edition of the World Health Organization (WHO) Classification of Breast Tumors are classified as "neuroendocrine tumor" and "neuroendocrine carcinoma." Key changes are exclusion of special histologic types (solid papillary carcinoma and hyper cellular variant of mucinous carcinoma) and the inclusion of large cell neuroendocrine carcinoma ${ }^{2}$. There are few clinical studies on NEC, and the number of cases involved is always small. Several clinical studies suggested that the prognosis of NEC patients is better than that of $I D C^{3-5}$ or had no significant differences between the two cohorts ${ }^{6}$. But some studies showed that compared with IDC, NEC was a more aggressive tumor and had worse prognosis ${ }^{7-11}$. Data regarding the prognostic significance of NEC are conflicting in several studies ${ }^{12-13}$. 
The treatment of NEC is consistent with that of invasive breast cancer. Clinicians knew little about NEC and usually treated them according to IDC. We took advantage of a large database of cancer cases from surveillance, Epidemiology, and end results (SEER) registries. The aim of this study was to compare the clinic pathological features and clinical outcome between breast neuroendocrine carcinoma and the most common breast invasive ductal carcinoma (IDC) and to know the independent prognostic risk factors of NEC using SEER database.

\section{Methods}

\section{Patients}

The Surveillance, Epidemiology, and End Results (SEER) database from 1973 to 2015 was used to collect patients' clinic pathological features and survival data. Since the HER2 information was available after 2010 and there has been a study on SEER database between 2003 and 2009, we collected the SEER patients diagnosed between 2010 and 2015. Other selection criteria were as

Follows: the study cohort of NEC (ICD-0-3 8246/3, ICD-0-3 8041/3 and ICD-0-3 8013/3) and IDC (ICD-0-3 8500/3).

\section{Clinic pathological characteristics}

To investigate the clinic pathological characteristics of NEC and IDC of the breast, the following information was obtained: age, sex, race, grade, laterality, pathological type, AJCC stage, tumor size, nodal stage, metastasis status, breast molecular subtype, surgery treatment with either mastectomy or breast-conserving surgery, radiation therapy, chemotherapy, marital status at diagnosis, cause of death, and survival (months). There were only two patients with HR+/HER2+ left when we talk about independent prognostic factors after screening out unknown factors. And there were no population of type HR-/HER2 + in the collected cases, so we categorized the patients into two subtypes later: HR+ and triple negative breast cancer (TNBC).

\section{Statistical analyses}

Clinic pathological characteristics were compared between NEC and IDC by Pearson chi-square and Fisher's exact probability tests. Kaplan-Meier plots and log-rank tests were performed to compare OS and BCS between the two cohorts. Propensity score matching was conducted to calibrate the effects of the baseline of clinic pathological differences. The univariate and multivariate Cox proportional hazard model was applied for identifying prognostic factors. All the statistical analyses and graphics were performed with the SPSS statistical software, version 22.0 (IBM Corp, Armonk, NY).

\section{Results}

\section{Patient characteristics}


In total, we identified 208 patients with breast NEC and 275,878 cases with breast IDC from SEER database between the years 2010 to 2015 in our study. The clinic pathological characteristics of the 208 NEC patients were compared with those of IDC, and the results are summarized in Table 1. There were significant differences between NEC and IDC, including Grade, Laterality, AJCC stage, T stage, N stage, M stage, molecular subtype, and treatment options. NEC tumors were significantly larger than IDC tumors $(p=0.000)$ (Table 1). The mean NEC size was $34 \mathrm{~mm}$, whereas the mean IDC size was $22 \mathrm{~mm}$. Compared with IDC, patients with NEC had higher grade(III-IV, 50.0\% vs. 38.3\%, $\mathrm{p}=0.000)$, higher stage (II-IV,75.0\% vs. $46.2 \%, p=0.000$ ), higher rate of distant metastasis ( $M 1,20.2 \%$ vs. $4.9 \%, p=0.000$ ). There were lower patients with HER2 positive in NEC cohort than IDC cohort (HER2+, $1.9 \%$ vs. $16.1 \%, p=0.000$ ). Concerning treatment options, more NEC patients chose not to undergo surgery (35.1\% vs. 9.0\%), and fewer patients chose breast conserving surgery than IDC patients $(33.2 \%$ vs. $51.1 \%, p=0.000)$. Compared with IDC patients, NEC patients were more likely to choose chemotherapy ( $48.1 \%$ vs. $41.0 \%, p=0.039)$ while less likely to receive radiotherapy ( $34.1 \%$ vs. $46.3 \%, p=0.000)$. Since the clinic pathological characteristics of NEC and IDC were significantly different which may influence the clinical outcome, we performed a 1:4 (NEC/IDC) propensity score matching(PSM) analysis to the utmost to eliminate the baseline variations(Figure S1 and S2). After excluding patients with unknown baseline characteristics, there were 132 patients left in NEC cohort. After PSM analysis, we had 130 cases in NEC cohort and 531 cases in IDC cohort. The results of comparison of NEC and IDC clinic pathological features are summarized in Table 1. The baseline of NEC and IDC was well balanced after PSM.

\section{Survival analyses}

NEC showed a significantly worse clinical outcome than breast IDC by the Kaplan-Meier plot (Fig. 1, both $\mathrm{p}<0.001$ ). The 66-month overall survival (OS) rate in NEC and IDC was $44.5 \%$ and $80.2 \%$, respectively. The 66-month breast cancer specific survival (BCSS) rate in NEC and IDC was $56.3 \%$ and $88.8 \%$, respectively. NEC showed a poorer clinical outcome than IDC after PSM. The median follow-up time in NEC cohort was 25 months (interquartile range [IQR], 10.75-38.50 months) while in IDC cohort was 27 months (interquartile range [IQR], 12-48 months). NEC had a worse OS $(p=0.002)$ and BCSS $(p=0.001)$ compared with IDC (Fig. 2). The 66-month OS rate in NEC and IDC was $49.8 \%$ and $71.4 \%$ after PSM, respectively. And the 66 -month BCSS rate in NEC and IDC was $54.1 \%$ and $80.5 \%$ after PSM, respectively.

\section{Prognostic factors}

In order to obtain the independent risk factors affecting the clinical outcome of NEC, we performed univariate Cox analysis and multivariate Cox analysis. The results of univariate Cox analysis was shown in the Table 2. According to the univariate Cox results and clinical experience, we included age, NEC pathological type, AJCC stage, T stage, $\mathrm{N}$ stage, M stage, operation mode, radiotherapy, chemotherapy and molecular typing into multivariate Cox analysis. We found that the independent risk factors affecting the prognosis of NEC were age, pathological type, stage, M stage, chemotherapy and molecular typing for OS, while age, pathological type, M stage, chemotherapy and molecular typing for BCSS (Table 3). Patients who are $\geq 60$ years old, with high AJCC stage, with distant metastases, with special pathological 
type (large cell neuroendocrine carcinoma and small cell carcinoma), with the molecular typing of TNBC and without chemotherapy had worse clinical outcome. We made a monogram for OS in breast NEC (Figure 3, c-index=0.835).

\section{Therapeutic Modalities}

As is shown in Table 4, chemotherapy was the independent risk factor for the clinical outcome of NEC while surgery mode and radiation made no influences. Considering the close relationship between surgery and radiotherapy, we made a comparison of interactive factors (Table 4). It seemed that patients with the therapeutic modalities of mastectomy + no radiation and breast-conserving surgery + radiation had better OS and BCSS. Considering that the choice of operation mode is closely related to the stage TNM, age, subtype and chemotherapy of patients, we adjusted hazard ratio in order to exclude the influence of these factors. The therapeutic modalities of surgery mode and radiation had no effect on the prognosis of NEC after adjusted.

\section{Discussion}

Neuroendocrine carcinoma of the breast is a rare disease which is not well understood by many clinicians. There were 142 SEER cases between the years 2003 and 2009 reported in Wang's article in $2014^{10}$ which was the study with the largest number of NEC cases. Because Her-2 is an important molecular subtype factor and this information is only available after 2010, we collected the SEER patients diagnosed between 2010 and 2015. Also, we included large cell neuroendocrine carcinoma which was newly added into the neuroendocrine breast neoplasms in the 5th Edition of the World Health Organization (WHO). At present, only 7 cases of large cell neuroendocrine carcinoma of the breast have been reported ${ }^{14-20}$. Finally, 208 patients with breast NEC were identified in the present study.

In our study, we found NEC has distinct clinic pathological features. Compared with IDC, NEC had higher stage, larger tumor size and more distant transfers. We found that the majority of NEC patients were found at a higher stage, which may be due to the lack of specific imaging and clinical manifestations ${ }^{21}$. The mean age of NEC patients was 63 years old in our study, which was consistent with the information that most patients are postmenopausal in other studies ${ }^{22-25}$. There were only 4 patients with HER2 + in our study, and most of patients were HR + / HER2 -. The results of subtype were consistent with previous studies $^{6,26-27}$. The 66 -month overall survival (OS) rate in NEC was $44.5 \%$ and $56.3 \%$, which was worse than IDC, respectively. The poor clinic outcome may be due to its aggressive clinic pathological features. In order to eliminate the impact of the baseline, we performed a 1:4 (NEC/IDC) propensity score matching (PSM) analysis. After PSM, there were still significant differences on OS and BCSS of NEC and IDC.

This poor clinical outcome was similar to previous reports but also contrary to the results of some articles. Rovera et al. ${ }^{5}$ analyzed 96 NEC patients in 1992-2013 and found their 10-year overall survival was $87 \%$, which was significant different from our study. Lopez-Bonet et al. ${ }^{3}$ reported 7 cases of breast NEC and none of them died from BC-related causes after a median follow-up of 51 months. Roininen et 
al. ${ }^{7}$ collected 43 NEBC cases from Oulu and Helsinki University Hospitals in 2007-2015 and reported that relapse-free survival $(p=0.001)$, disease-free survival $(p=0.024)$ and overall survival $(p=0.003)$ of IDC was better compared to NEC. Zhang et al. ${ }^{8}$ analyzed 107 Chinese patients with breast NEC and found NEC was more likely to have local recurrence and poor overall survival. They also found T, M, AJCC stage and Ki67 were independent prognostic factor of the clinic outcome. Wei B et al. ${ }^{9}$ collected 68 NEC patients from the University of Texas M. D. Anderson Cancer Center and found high nuclear grade, large tumor size and regional lymph node metastasis were significant negative prognostic factors for overall survival. The independent risk factors affecting the prognosis of NEC are not well established now ${ }^{13}$.

In our study, we analyzed 132 NEC patients with complete data and found the independent risk factors affecting the prognosis of NEC were age, pathological type, AJCC stage, $\mathrm{M}$, chemotherapy and molecular typing for OS, while age, pathological type, $\mathrm{M}$, chemotherapy and molecular typing for BCSS. Patients with special pathological type of NEC, large cell neuroendocrine carcinoma and mall cell carcinoma, had worse OS $(p=0.012)$ and BCSS $(p=0.027)$. Patients with NEC can benefit from chemotherapy $(H R=$ $8.468, p=0.000$ ) which is the only factor that clinicians can control. At present, doctors determine whether patients need chemotherapy and chemotherapy regimens according to the patient's age, molecular typing, stage, 21 gene and so on ${ }^{26,28}$. We suggest NEC patients all receive chemotherapy if it is necessary. For the choice of surgery and radiotherapy, there were no significant impacts on the prognosis after correction in our study. We think that doctors may choose the appropriate surgical and radiotherapy methods according to the current guidelines without special preference according to our results. While in Richter-Ehrenstein's article, mastectomy is more suitable for NEC than breast conserving surgery due to its early invasion potential. More studies should be performed to analyze the surgical procedures and radiotherapy.

The present study had several limitations. Recurrence free survival rate is an important information to evaluate the invasiveness of a tumor. Due to a lack of recurrence information in SEER database, our study didn't analyze the recurrence free survival rate. Also, as in shown in previous, Ki67 may influence the clinic outcome and is an important clinic pathological feature, which has not been recorded in SEER database. In addition, some bias may occur due to the small sample size. For example, there were only 2 male patients included in the prognostic factor analysis. The SEER database also lacks the information

of endocrine therapy and the specific regimen of chemotherapy. According to the literature data ${ }^{26}$, cisplatin and etoposide are used in small cell carcinoma, which is different from the chemotherapy regimen of non-small cell carcinoma. Therefore, a further expanded study is warranted to verify our findings.

\section{Conclusion}

We showed that breast NEC has distinct clinic pathological features and it is a rare tumor with stronger invasion and significantly worse clinic outcome compared with breast IDC. The independent risk factors 
affecting the prognosis of NEC were age, pathological type, AJCC stage, M, chemotherapy and molecular typing. Surgery mode and radiation therapy made no influence on the clinic outcome of NEC.

\section{Declarations}

\section{Acknowledgements}

The authors acknowledge the efforts of the Surveillance, Epidemiology, and End Results (SEER) Program tumor registries in the creation of the SEER database.

\section{Author contributions}

Chen, Xue, Xu contributed to the concept and design of the study. Wang, Huo contributed to the analysis and interpretation of data, and the drafting of the article. All authors contributed to the revision, approved the final version to be published, and agree to be accountable for all aspects of the work.

\section{Funding}

This work was supported by the research fund from science and technology projects in Jiangsu Province of China (Grant Number: BE2017726).

\section{Availability of data and materials}

All data generated or analyzed during this study are included in this published article [and its sementary information files].

\section{Ethics approval and consent to participate}

This article does not contain any studies with human participants or animals performed by any of the authors.

\section{Consent for publication}

Not applicable.

\section{Competing interests}

The authors declare that they have no competing interests.

\section{Author details}

${ }^{1}$ Affiliated Jinling Hospital, Medical School of Nanjing University, Nanjing 210002, China

${ }^{2}$ Research Institute of General Surgery, Nanjing Jinling Hospital, 305 Zhongshan East Road, Nanjing 210002 , China 
${ }^{3}$ Clinical pharmacology, Nanjing Jinling Hospital, 305 Zhongshan East Road, Nanjing 210002,

${ }^{4}$ China Southeast University, Nanjing, China

${ }^{5}$ Nanjing Medical University, Nanjing, China

\section{References}

(1). Xiang DB, Wei B, Abraham SC, et al. Molecular cytogenetic characterization of mammary neuroendocrine carcinoma. Human Pathology, 2014, 45(9):1951-1956.

(2). WHO Classification of Tumors Editorial Board. Breast Tumours. WHO Classification of Tumors, 5th edn. Lyon, France: IARC; 2019.

(3). Menendez. Solid neuroendocrine breast carcinomas: incidence, clinico-pathological features and immunohistochemical profiling. Oncol Rep, 2008, 20(6) :1369-1374.

(4). Righi L, Sapino A, Caterina Marchiò, et al. Neuroendocrine differentiation in breast cancer: established facts and unresolved problems.[J]. Seminars in Diagnostic Pathology, 2010, 27(1):69-76.

(5). Rovera F, Lavazza M, Rosa SL, et al. Neuroendocrine breast cancer: retrospective analysis of 96 patients and review of literature. International Journal of Surgery, 2013, 11:S79-S83.

(6). Bogina G, Munari E, Brunelli M, et al. Neuroendocrine differentiation in breast carcinoma: clinicopathological features and outcome. Histopathology, 2016, 68(3):422-432.

(7). Roininen N, Takala S, Haapasaari KM, et al. Primary neuroendocrine breast carcinomas are associated with poor local control despite favourable biological profile: a retrospective clinical study. BMC Cancer, 2017, 17(1): 72.

(8). Zhang Y, Chen Z, Bao Y, Du Z, Li Q, Zhao Y, et al. Invasive neuroendocrine carcinoma of the breast: a prognostic research of 107 chinese patients. Neoplasma, 2013, 60(02), 215-222.

(9). Wei B, Ding T, Xing Y, Wei Wei MS, Tian Z, Tang, F, et al. Invasive neuroendocrine carcinoma of the breast. Cancer, 2010, 116(19), 4463-4473.

(10). Wang J, Wei B, Albarracin CT, Hu J, Abraham SC, Wu Y. Invasive neuroendocrine carcinoma of the breast: a population-based study from the surveillance, epidemiology and end results (SEER) database. BMC Cancer, 2014, 14:147.

(11). Kwon SY, Bae YK, Gu MJ, et al. Neuroendocrine differentiation correlates with hormone receptor expression and decreased survival in patients with invasive breast carcinoma. Histopathology, 2014, 64(5):647-659. 
(12). Tian Z, Wei B, Tang F, et al. Prognostic significance of tumor grading and staging in mammary carcinomas with neuroendocrine differentiation. Hum Pathol, 2011, 42(8):1169-1177.

(13). Pareja F, D'Alfonso TM. Neuroendocrine neoplasms of the breast: A review focused on the updated World Health Organization (WHO) 5th Edition morphologic classification. Breast J, 2020, 26(6):11601116.

(14). Safini F, Bouchbika Z, Bennani Z, et al. Primary large cell neuroendocrine carcinoma of the breast: a rare tumor in humans. Pan Afr Med J, 2016, 25():205.

(15). Yoshimura N, Sasada T, Yonehara S. Primary Large-Cell Neuroendocrine Carcinoma of the Breast Occurring in a Pre-Menopausal Woman. Breast Care (Basel), 2015, 10(4):281-283.

(16). Janosky M, Bian J, Dhage S, et al. Primary large cell neuroendocrine carcinoma of the breast, a case report with an unusual clinical course. Breast J, 2015, 21(3):303-307.

(17). Omachi N, Shimizu S, Kawaguchi T, et al. A case of large-cell neuroendocrine carcinoma harboring an EML4-ALK rearrangement with resistance to the ALK inhibitor crizotinib. J Thorac Oncol, 2014, 9(6):e40-42.

(18). Psoma E, Nikolaidou O, Stavrogianni T, et al. A rare case report of a primary large-cell neuroendocrine carcinoma of the breast with coexisting Paget disease. Clin Imaging, 2012, 36(5):599601.

(19). Okoshi K, Saiga T, Hisamori S, et al. A case of cytokeratin 20-positive large-cell neuroendocrine carcinoma of the breast. Breast Cancer, 2012, 19(4):360-364.

(20). Kim JW, Woo OH, Cho KR, et al. Primary large cell neuroendocrine carcinoma of the breast: radiologic and pathologic findings. J Korean Med Sci, 2008, 23(6):1118-1120.

(21). Park YM, Wu Y, Wei W, Yang WT. Primary neuroendocrine carcinoma of the breast: Clinical, imaging, and histologic features. AJR Am. J. Roentgenol, 2014, 203(): W221-W230.

(22). Cloyd JM, Yang RL, Allison KH, et al. Impact of histological subtype on long-term outcomes of neuroendocrine carcinoma of the breast. Breast Cancer Res Treat, 2014, 148():637-644.

(23). Roininen N, Takala S, Haapasaari KM, et al. Primary neuroendocrine breast carcinomas are associated with poor local control despite favourable biological profile: a retrospective clinical study. BMC Cancer, 2017, 17:72.

(24). Kelten Talu C, Leblebici C, Kilicaslan Ozturk T, et al. Primary breast carcinomas with neuroendocrine features: clinicopathological features and analysis of tumor growth patterns in 36 cases. Ann Diagn Pathol, 2018, 34:122-130. 
(25). Lavigne M, Menet E, Tille JC, et al. Comprehensive clinical and molecular analyses of neuroendocrine carcinomas of the breast. Mod Pathol, 2018, 31:68-82.

(26). Abdelwahed A, Ahmed M. Rare epithelial breast cancer: Surgery and adjuvant therapy. Transl. Cancer Res, 2019, 8: S479-S492.

(27). Kawasaki T, Mochizuki K, Yamauchi H, et al. High prevalence of neuroendocrine carcinoma in breast lesions detected by the clinical symptom of bloody nipple discharge. Breast, 2012, 21(5):652-656.

(28). Richter-Ehrenstein C, Arndt J, Buckendahl AC, Eucker J, Weichert W, Kasajima A, Schneider A, Noske A. Solid neuroendocrine carcinomas of the breast: Metastases or primary tumors? Breast Cancer Res. Treat, 2010, 124, 413-417.

\section{Tables}

Due to technical limitations, table 1-4 is only available as a download in the Supplemental Files section.

\section{Figures}

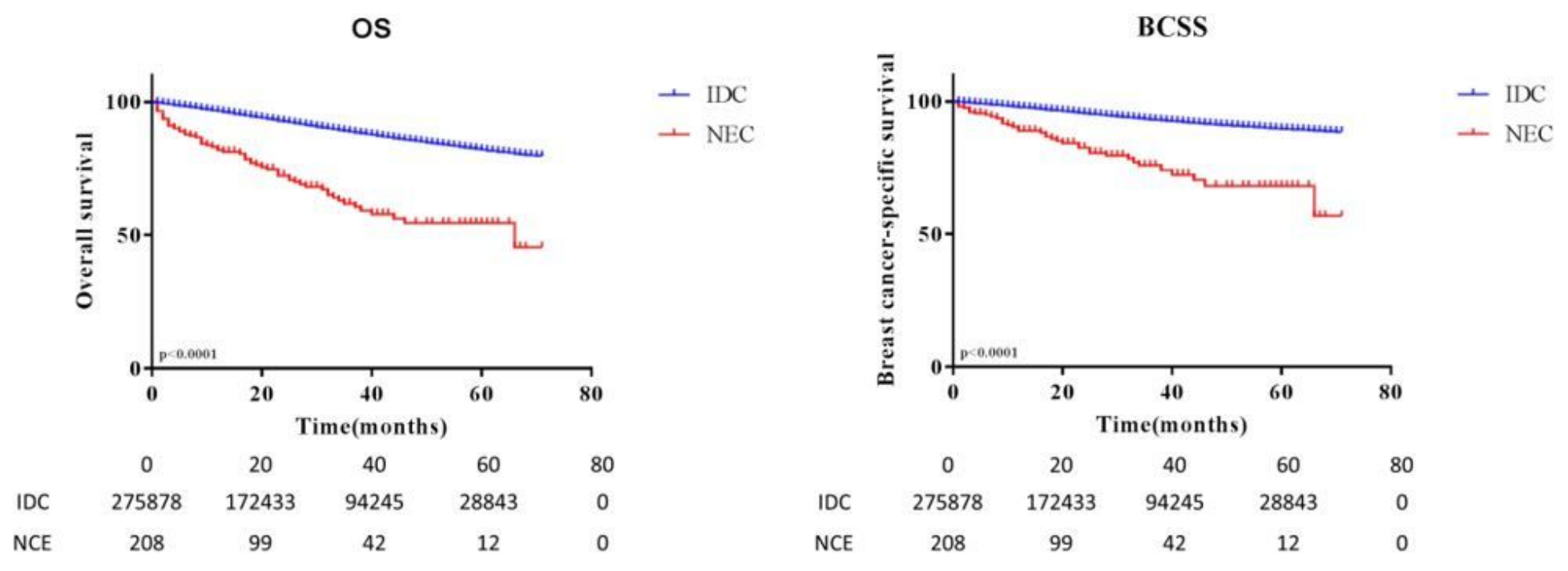

Figure 1

Kaplan-Meier curves showing a comparison of OS (a) and BCSS (b) between invasive ductal carcinoma (IDC) and neuroendocrine carcinoma (NEC). 

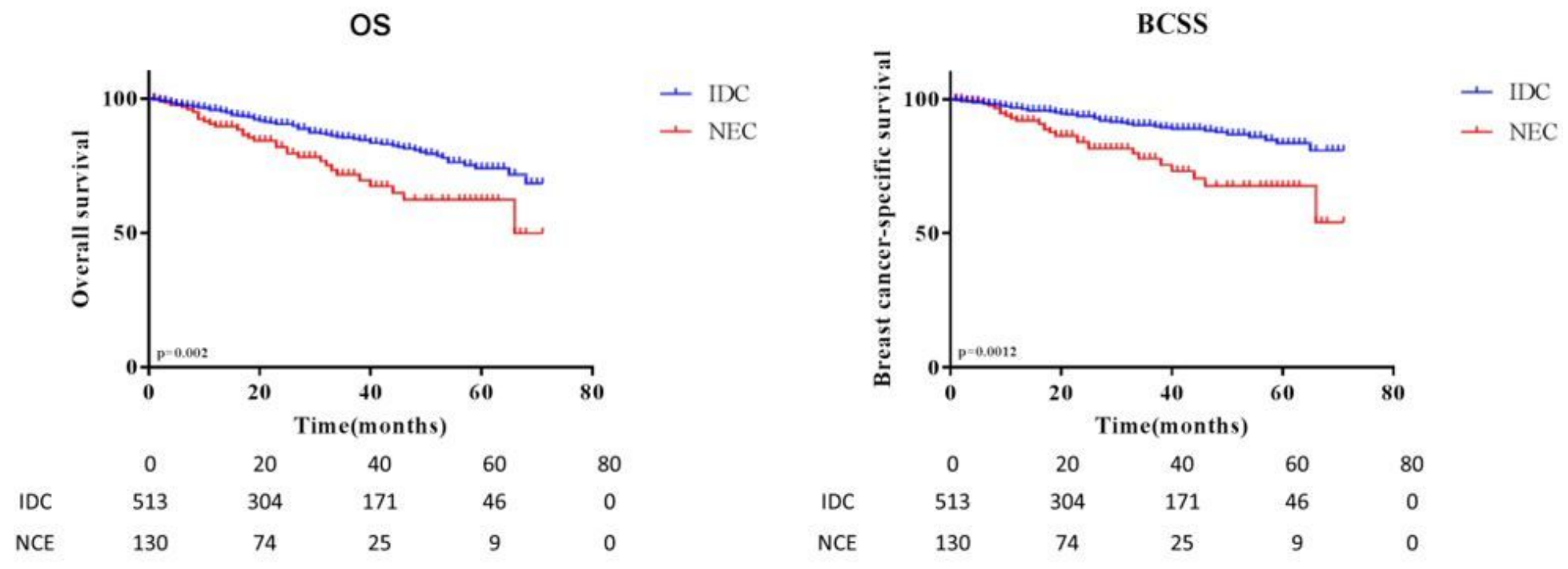

Figure 2

Kaplan-Meier curves showing a comparison of OS (a) and BCSS (b) between invasive ductal carcinoma (IDC) and neuroendocrine carcinoma (NEC) after PSM.

Points

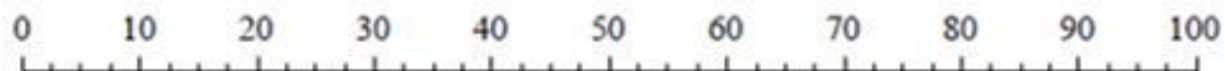

Age

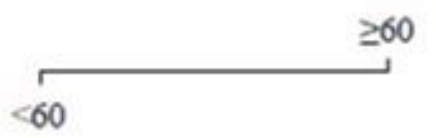

Stage

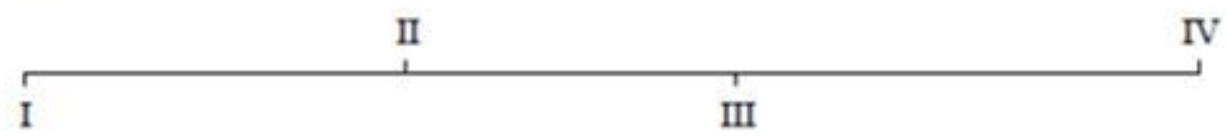

Cheomtherapy ${ }_{\text {Yes }}^{\text {No }}$

Subtype

TNBC

HR+

ICDO

Small cell carcinoma

Neuroendocrine carcinoma Large cell neuroendocrine carcinoma

Total points

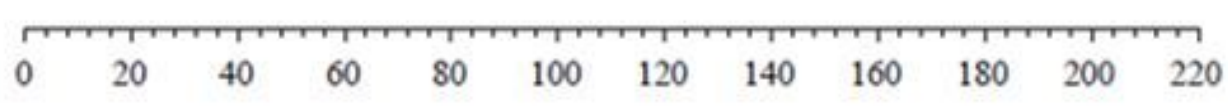

3-Year survival

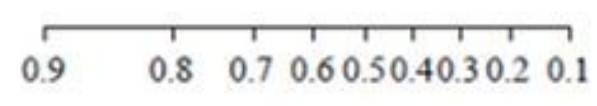

5-Year survival

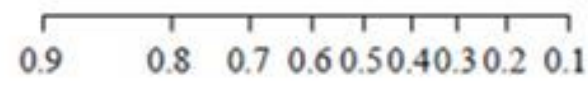


Figure 3

OS monograms of breast NEC. M and stage have a dominant relationship, so only one is taken as the variable. When $M$ is used, $c$-index $=0.811$. When Stage is used, $c$-index $=0.835$.

\section{Supplementary Files}

This is a list of supplementary files associated with this preprint. Click to download.

- Table14.pdf

- FigS1.JPG

- Figs2.JPG 